\title{
A Roadmap to Inform Development, Validation and Approval of Digital Mobility Outcomes: The Mobilise-D Approach
}

\author{
Lynn Rochester ${ }^{a, b} \quad$ Claudia Mazzàc, d Arne Muellere \\ Brian Caulfield ${ }^{\mathrm{f} g} \quad$ Marie McCarthy $^{\mathrm{h}} \quad$ Clemens Becker $^{\mathrm{i}}$ Ram Miller ${ }^{\mathrm{e}}$ \\ Paolo Pirainoj Marco Vicecontik Wilhelmus P. Dartee ${ }^{l}$ \\ Judith Garcia-Aymerich ${ }^{m-0}$ Aida A. Aydemir ${ }^{p}$ Beatrix Vereijken ${ }^{q}$ \\ Valdo Arnerar ${ }^{r}$ Nadir Ammour ${ }^{s}$ Michael Jackson ${ }^{t}$ Tilo Hache ${ }^{e}$ \\ Ronenn Roubenoffe on behalf of the Mobilise-D Consortium \\ ${ }^{a}$ Translational and Clinical Research Institute, Newcastle University, \\ Newcastle upon Tyne, UK; ${ }^{b}$ The Newcastle upon Tyne NHS Foundation Trust, \\ Newcastle upon Tyne, UK; ${ }^{c}$ Department of Mechanical Engineering, The University of \\ Sheffield, Sheffield, UK; dINSIGNEO Institute for in Silico Medicine, The University of \\ Sheffield, Sheffield, UK; e Translational Medicine, Novartis Institutes for Biomedical \\ Research, Basel, Switzerland; Insight Centre for Data Analytics, University College Dublin, \\ Dublin, Ireland; ${ }^{9}$ School of Public Health, Physiotherapy and Sports Science, University \\ College Dublin, Dublin, Ireland; ${ }^{\mathrm{h} I C O N}$ plc., Dublin, Ireland; 'Robert Bosch Foundation for \\ Medical Research, Stuttgart, Germany; ${ }^{j}$ Research and Early Development Statistics, Bayer, \\ Berlin, Germany; 'University of Bologna, Bologna, Italy; 'GDD Regulatory Affairs, Novartis \\ Pharma AG, Basel, Switzerland; ${ }^{m}$ ISGlobal, Barcelona, Spain; ${ }^{n}$ Universitat Pompeu Fabra (UPF), \\ Barcelona, Spain; ${ }^{\circ}$ CIBER Epidemiología y Salud Pública (CIBERESP), Madrid, Spain; \\ PEMD Serono, Billerica, MA, a Business of Merck KGaA, Darmstadt, Germany; \\ 9Department of Neuromedicine and Movement Science, Norwegian University of \\ Science and Technology, Trondheim, Norway; 'ERT Geneva, Geneva, Switzerland; ${ }^{\text {s} S a n o f i ~}$ \\ R\&D, Clinical Sciences and Operations, Chilly-Mazarin, France; ${ }^{t}$ iXscient Ltd. (IXS), \\ London, UK
}

Keywords

Remote Monitoring · Body-worn devices · Digital mobility outcomes

\section{Abstract}

Health care has had to adapt rapidly to COVID-19, and this in turn has highlighted a pressing need for tools to facilitate remote visits and monitoring. Digital health technology, including body-worn devices, offers a solution using digital outcomes to measure and monitor disease status and provide outcomes meaningful to both patients and health care professionals. Remote monitoring of physical mobility is a prime example, because mobility is among the most advanced modalities that can be assessed digitally and remotely. Loss of mobility is also an important feature of many health conditions, providing a read-out of health as well as a target 
for intervention. Real-world, continuous digital measures of mobility (digital mobility outcomes or DMOs) provide an opportunity for novel insights into health care conditions complementing existing mobility measures. Accepted and approved DMOs are not yet widely available. The need for large collaborative efforts to tackle the critical steps to adoption is widely recognised. Mobilise-D is an example. It is a multidisciplinary consortium of 34 institutions from academia and industry funded through the European Innovative Medicines Initiative 2 Joint Undertaking. Members of Mobilise-D are collaborating to address the critical steps for DMOs to be adopted in clinical trials and ultimately health care. To achieve this, the consortium has developed a roadmap to inform the development, validation and approval of DMOs in Parkinson's disease, multiple sclerosis, chronic obstructive pulmonary disease and recovery from proximal femoral fracture. Here we aim to describe the proposed approach and provide a high-level view of the ongoing and planned work of the Mobilise-D consortium. Ultimately, Mobilise-D aims to stimulate widespread adoption of DMOs through the provision of device agnostic software, standards and robust validation in order to bring digital outcomes from concept to use in clinical trials and health care.

(C) 2020 The Author(s)

Published by S. Karger AG, Basel

\section{Introduction}

Physical mobility is important. In fact, it is fundamental to life. Most of us take it for granted and never experience the consequences of immobility through ill health. COVID-19 however has given us a sharp reminder of how important it is. The consequence of immobility - imposed externally through lockdown rather than internally by disease - is being revealed layer by layer at a personal and societal level, starting from the experience of restricted mobility and freedom, to the evolving impact on physical and mental health. The effects of COVID-19 are being felt disproportionately among older adults especially those with chronic ill health and multimorbidity $[1,2]$.

Health care has had to rapidly adapt to the pandemic with virtual clinics the "new normal" thanks to removal of barriers previously seen as insurmountable (data security, privacy, data breaches, patient acceptability, insurance coverage). Clinical research is facing similar challenges requiring rapid protocol and study delivery changes to ensure viability. The tools to facilitate remote visits and monitoring have never been more necessary. Digital health technology, including body-worn devices, offers a way forward by using digital outcomes to measure and monitor disease status and clinical outcomes meaningful to patients and health care professionals.

Remote monitoring of physical mobility is a prime example of what the future may look like, because mobility is among the most advanced modalities being assessed digitally and remotely. Loss of mobility is also an important feature of many health conditions, providing a read-out of health as well as a target for intervention [3,4]. Real-world digital measures of mobility are therefore an area of promise for remote monitoring of health care conditions. In the last decade, advances in sensor technology have led to sensors that are able to record continuously for longer periods of time (weeks and months) and include multiple capabilities. This opens the opportunity to extend the scope of mobility measurement to remotely and continuously capture discrete, relevant and ecologically valid mobility characteristics (such as real-world walking speed and life space). This digital approach to mobility assessment allows measurement to be performed anywhere and anytime providing an inclusive approach for patients.

Although digital mobility assessment with wearable sensors offers a solution for remote monitoring of digital mobility outcomes, widely accepted tools are not yet available [5]. The 
use of digital health technologies in clinical trials and ultimately health care poses significant challenges because of the multiple types of expertise (e.g., technical, clinical and regulatory) and steps (including both technical and clinical validation) required to deliver the evidence needed for adoption. Large collaborative consortia are recognised as an essential part of an overall strategy to develop and adopt digital health technologies and digital biomarkers in clinical trials [6].

Mobilise-D (Connecting digital mobility assessment to clinical outcomes for regulatory and clinical endorsement, www.mobilise-d.eu) is an example of one of the leading efforts aiming to deliver a solution for remote digital assessment of mobility. Mobilise-D is a publicprivate partnership funded by the European Innovative Medicines Initiative 2 Joint Undertaking. The Mobilise-D consortium includes 34 international research partners based at leading universities and some of the world's largest pharmaceutical and technical companies. The overarching objective of Mobilise-D is to obtain regulatory approval for digital mobility outcomes in a variety of disease states - Parkinson's disease (PD), chronic obstructive pulmonary disease (COPD), multiple sclerosis (MS) and recovery from proximal femoral fracture (PFF). The consortium will carry out a 5-year programme of activity (which commenced in April 2019) towards this objective. Importantly, this work takes place in a precompetitive context which is critical for success. A guiding principle of the collective efforts of the consortium is transparency aided by ongoing and regular interaction with external stakeholders. To ensure an enduring legacy, data will be accessible through a digital data biobank, and algorithms will be developed in an agnostic and fully documented manner for the wider benefit of developers.

Aims

In this paper, we aim to share the strategy developed and adopted by the Mobilise-D consortium and provide a high-level view of the ongoing work of Mobilise-D. We share the roadmap used to inform the development, validation and approval of digital mobility outcomes (DMOs), identify the challenges and provide an overview of the critical steps to bring digital biomarkers from conception to adoption.

\section{Challenges to Adoption of DMOs}

For digital health technologies and DMOs to be adopted, considerable multidisciplinary efforts are needed [6]. Important contributions have been made to bring stakeholders onto the same page, e.g. harmonisation of terms for biomarkers and end points [7], guidelines for development of novel digital end points $[8,9]$, frameworks for validation and primers [1012], and guidelines for interactions with regulators [13-15]. However, the challenge for anyone aiming to deliver digital tools approved for use in research and ultimately clinical practice is to bring these recommendations together in one seamless roadmap from inception to adoption. The Mobilise-D consortium aims to learn from and adopt these best practices whilst innovating in this space - leading with development of standards, disease and hardware agnostic approaches, open access tools and databases amongst other things. Technical and clinical validation, evaluation of acceptability and stakeholder engagement are undertaken within a multidisciplinary framework of collaboration and transparency. Figure 1 sets out the critical steps towards adoption. Throughout, embedded stakeholder engagement, external scientific advisory input and patient and public involvement will enrich the work of the Mobilise-D consortium.

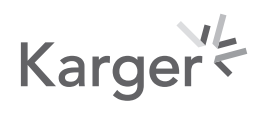




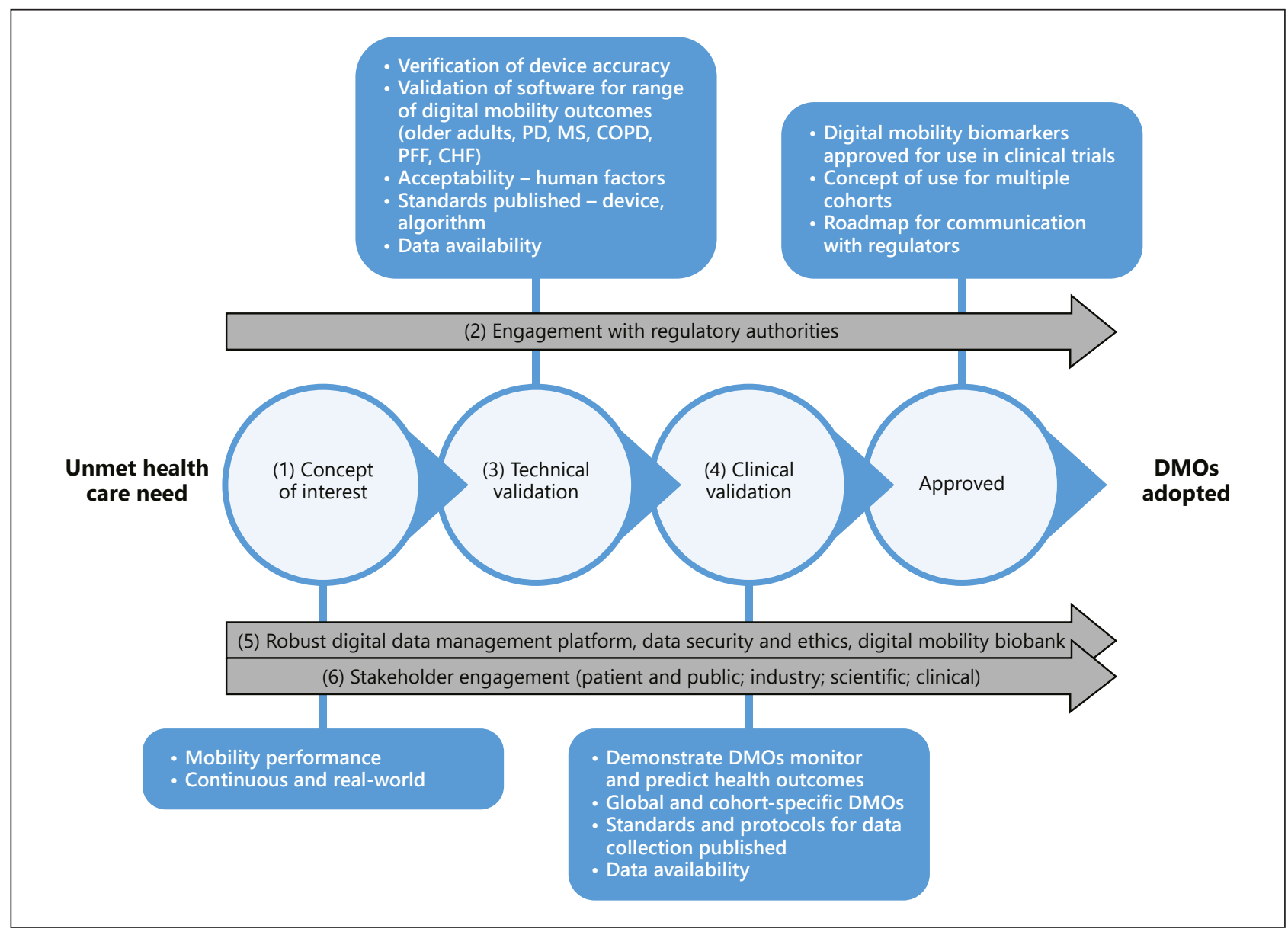

Fig. 1. The Mobilise-D roadmap. The critical steps of Mobilise-D from concept to adoption and their supporting work streams are illustrated and identified numerically. The anticipated outputs are described in the boxes for each critical step. The numbers correspond to the sections in the text "Critical steps in Mobilise-D to bring validated DMOs to regulatory approval." DMO, digital mobility outcome; PD, Parkinson's disease; MS, multiple sclerosis; COPD, chronic obstructive pulmonary disease; PFF, proximal femoral fracture; CHF, congestive heart failure.

\section{Critical Steps in Mobilise-D to Bring Validated DMOs to Regulatory Approval}

The following section describes the challenges and critical steps to achieve the objectives of the Mobilise-D consortium (summarised in Fig. 1). For clarity, a DMO is defined as a mobility outcome quantified from a wearable device (inertial measurement unit - IMU), using algorithms to extract specific mobility features of interest. In Mobilise-D, we are specifically interested in DMOs collected continuously during everyday walking activities. We adopt a comprehensive approach to quantify DMOs harmonised across our cohorts (Table 1). Systematic reviews of the literature (in progress) will provide supporting evidence for these DMOs [16]. For simplicity we use the term DMO to be inclusive, because the evidence to identify the optimal measure is a key part of our ongoing validation studies and reviews. Walking speed is used as an example of a measure for illustrative purposes because of extensive work with this outcome under controlled conditions. However, the Mobilise-D consortium will not limit validation to a single DMO. 
Table 1. Examples of digital mobility outcome measures selected for validation in Parkinson's disease, multiple sclerosis, chronic obstructive pulmonary disease and proximal femoral fracture recovery

Average walking speed over a rectilinear walking bout $(\mathrm{m} / \mathrm{s})$

Metrics extracted for each walking bout

- Cadence (steps/min)

- Step/stride time (s)

- Swing/stance time (s)

- Stride length (m)

- Turn duration (s)

- Turn angle (degrees)

- Turn velocity (mean and peak) (degrees/s)

Metrics extracted from all walking bouts (at daily level)

- Volume of walking: number, length and duration of walking bouts

- Number of steps per day, walking time, walking/movement intensity

\section{Mobility - Is It a Global Concept of Interest?}

Mobility is a term that describes the ability to move about in the home and community. Ageing and most chronic diseases make people physically weaker, less mobile and therefore less able to live independently. Walking is the commonest and most functionally relevant aspect of mobility affected by ageing and multiple chronic diseases. Walking requires cardiovascular, pulmonary and musculoskeletal systems as well as widespread brain networks for effective performance [17]. Changes in any of these systems because of ageing and/or disease are reflected in walking performance. As such, walking speed is often considered the 6th vital sign of health [4] and represents an appropriate mobility measure for multiple populations. Strong evidence that mobility outcomes, such as walking speed and walking activity, predict morbidity, mortality, falls, cognitive impairment and disability [3, 18-20] provide further support for this contention. Mobility is therefore an important target for interventions across many diseases and is also highly relevant because it is an important read-out of the integrity of the brain and body.

Despite this, inconsistent testing procedures and wide variations in so-called "norms" have prevented walking outcomes from being widely adopted. Current methods to measure mobility have some important limitations. Mobility is often estimated using brief functional tests that have limited validity. For example, they can lead patients to over-perform in a short assessment. Furthermore, patients are required to attend a centre for assessment which reduces generalisability of study findings and overall quality of care. Better ways to detect and objectively measure mobility in real-world settings (and eventually capture early mobility problems) are needed that in turn will help accelerate development and testing of new treatments and lead to better tools for use in health care.

In our cohorts, mobility is estimated using brief functional tests such as the 6-min walking test [21], the 10-metre walking test [22], the 25-foot walk [23], the "timed-up-and-go test" [24] or the 4-metre walk test as part of the Short Physical Performance Battery [25]. Consequently, walking assessment is inconsistent within and between diseases, precluding a harmonised approach to the measurement and understanding of mobility disability. A further shortcoming of these tests is that they estimate capacity for mobility and therefore lack ecological validity; the fact that patients can move, does not necessarily mean that they do so in their day-to-day life [26-29]. There is therefore a strong argument for mobility assessment to reflect the real-world because this represents what a patient does do, not what they can do.

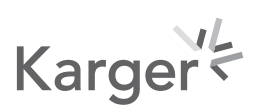


The Mobilise-D consortium describes mobility using 3 distinct constructs:

- Mobility capacity (what a person can do), which represents the patient's ability to move, and is provided by supervised clinical tests such as the 6-min walking test

- Mobility perception (what a person thinks he or she does), which represents the patient's subjective experience or perception of their own mobility, as provided through patientreported outcomes or clinician-assigned scores based on the patient's answers to standardised questionnaires, such as MDS-UPDRS-II [30], the MSWS-12 [31] or the PPAC [32]

- Mobility performance (what a person actually does) which represents the duration, quality and intensity of the participant's mobility as observed in real-world settings, during sufficiently long observational periods of time to be considered representative of daily life [33]. There are currently no accepted DMOs to quantify mobility performance

By taking this approach, DMOs collected continuously in the real-world complement existing mobility measures. Furthermore, the cohorts in Mobilise-D have very different traditional mobility outcomes, mobility difficulties and diverse disease trajectories, thereby allowing a comprehensive approach to quantify disability [34]. Ultimately, we see value in regulatory approval and health and technology assessment processes having a routine expectation for an accurate measurement of mobility under real-world circumstances. Mobility per se is therefore a relevant concept of interest for our cohorts and forms the rationale for the work of the Mobilise-D consortium (Fig. 1).

\section{Engaging the Regulators}

The Mobilise-D consortium aims to present five different contexts of use for DMOs to quantify mobility performance. Four will address each individual cohort (PD, COPD, MS and PFF), and one will propose a disease-independent DMO. Regulatory requirements and processes for DMOs differ depending on the context of use in which the DMO is to be used. The focus of Mobilise-D is the "qualification of a new methodology," which is where the DMO is used to inform the safety or efficacy of a new medical product, such as a new drug or digital therapeutic.

There are different strategies to pursue the regulatory approval of DMOs. For example, a DMO can be proposed as a clinical outcome (and therefore a primary clinical end point) in its own right to quantify mobility performance. However, changes in mobility are often an indirect outcome of a drug being tested, not the primary target. A second strategy can therefore be adopted that is more conservative where mobility is not the target outcome for the drug being tested. Instead, mobility performance can be proposed as a secondary DMO (or digital mobility biomarker) to monitor the health condition or as a surrogate of an accepted clinical end point. This is especially relevant for some health conditions where mobility perception and/or capacity outcomes are already accepted for monitoring the state of the disease. This requires demonstration of both technical validity (to accurately measure) and clinical validity of the new biomarker by showing construct validity, predictive capacity and the ability to detect change.

To our knowledge, there are currently only 2 cases where DMOs have been qualified for use in regulatory drug trials: the qualification opinion of the European Medicine Agency (EMA, EU Regulator) on the use of on-stride velocity 95th centile measured with wearable sensors as a secondary end point in Duchenne Muscular Dystrophy [35]; and the EMA Qualification Opinion requested by the PROactive consortium on the use of DMOs by COPD specialists to assign a mobility-related clinician-reported outcome [36]. Both opinions are very narrow in scope in contrast to Mobilise-D which is targeting 4 different cohorts. When Trium Analysis Online GmbH submitted a letter of intent to the US regulator, the Food and Drugs Administration (FDA), proposing to use change in continuous real-world walking speed as a clinical end point in patients with MS, FDA advice suggested a dual element quali-

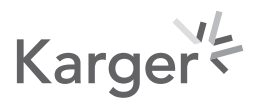


fication process, which requires the need to demonstrate not only the validity of the DMO, but also the necessity of developing this new clinical end point, which is usually a much more ambitious goal [37].

Our long-term goal is the qualification of DMOs for two contexts of use. The first is to use the DMO to monitor the progression of the disease, where mobility performance is reduced with disease progression. The second is to use DMOs as surrogates of disease-specific secondary end points such as falls in PD and MS. Following the recommendations of EMA experts [13], we engaged in very early dialogue and adopted a staged approach to regulatory qualification. First, we received a positive qualification advice on the validation protocol we proposed for the use of a DMO as an additional digital monitoring biomarker to account for mobility performance in assessing the efficacy of new or existing treatments for PD patients, complementing those already in use that account only for patients' perception of mobility and mobility capacity (see Viceconti et al. [38] for further detail). Second, we have recently requested a new qualification advice to find:

(i) the best DMO as a monitoring biomarker of mobility performance for each additional health condition (COPD, MS, and PFF);

(ii) the best DMO as a monitoring biomarker of mobility performance across all 4 health conditions;

(iii) the best DMO that can be used as a surrogate biomarker, to predict:

(a) for COPD: moderate-to-severe exacerbation requiring substantial changes in treatment and/or emergency room or hospital admission;

(b) for PFF: against admission to a long-term care facility or other form of assisted living setting;

(c) for MS and PD: against self-reported fall.

In all cases, the validation protocol we proposed includes an extensive technical validation with controlled experiments, and a clinical validation comprising the assessment of construct validity, predictive capacity and ability to detect change (described below). Key to this work was the need to engage in conversations with regulatory authorities at a very early stage, and this commenced at the start of Mobilise-D (Fig. 1) in parallel with the other activities of the consortium.

Technical Validation: Does It Do What It Says on the Tin?

While objectively measuring real-world mobility is very attractive, it is far from simple or straightforward. Objectively measuring the ability to move about in the home and community requires clear definitions of the mobility feature to be measured and validation in the environment, context and population for which it is intended. This raises a number of significant challenges (see Del Din et al. [39] for a review) that are being addressed in Mobilise$\mathrm{D}$ so that a robust device-agnostic solution to accurately measure real-world mobility, device standards and protocol standardisation can be achieved (Fig. 1).

An essential requirement for validation of DMOs is to have clear definitions of the aspect of mobility to be measured to define the requirements for algorithms. Take, for example, realworld walking speed as the DMO of interest. This requires that the precise start and end point of a "walking bout" is defined, and the timing and number of steps identified, from which the DMO is quantified. However, lack of consensus about what constitutes a walking bout, thresholds for bout length and the minimum number of steps required, has led to confusion and lack of consistency [39]. Brief pauses and resting periods between walking bouts [39-41] as well as turning $[42,43]$ driven by environmental contexts also affect the values of DMOs, especially when calculated as an average over a given walking bout [44, 45]. Mobilise-D has created a common language allowing algorithms to be developed according to a standard definition and parameters set consistently taking account of health condition and context.

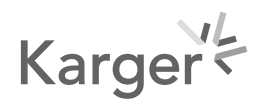


Algorithmic requirements within Mobilise-D include the ability to calculate the DMOs of interest in different daily life contexts and for different patient groups, which is particularly challenging for a single sensor solution. Algorithms will have to account for the huge variability of signal features, associated with intrinsic gait factors (e.g., slow and impaired gait pattern with various characteristics), contextual factors (e.g., environment, usage of various walking aids) and variations in protocol (location of IMU). Associated algorithmic challenges are being tackled combining state-of-the-art signal processing, activity recognition and labelling approaches, for which published algorithms will be selected according to performance on suitable existing data sets that have been made available to the consortium. Preprocessing approaches are being used to tackle filtering and enhancement of walking steprelated features [46]. Gait sequence detection is based on combining adaptive thresholding, frequency analysis, wavelet analysis and machine learning techniques [47-50]. Labelling of steps and strides is exploring time-domain [51], frequency-domain [52] and templatematching [53] approaches. All algorithms are being implemented using a sensor agnostic approach allowing them to be applied to new and existing data. Scripts are developed and fully documented on the GitLab platform, using an open-source license (GitLab Inc.). All the algorithms are run on the same standardised environment, and their executions are orchestrated by the open source e-Science Central platform [54]. Once optimised on pre-existing data, algorithms will be tested on new data from an ad hoc technical validation study.

Validation of DMOs against a gold standard system to verify accuracy of algorithm performance has to take context into account (real-world) [55]. This is relatively straightforward in the gait laboratory where sophisticated tools are available [56]. Observations conducted in a laboratory, however, are often limited to simple straight walking or to a range of activities mimicking daily life scenarios. Validation of a single sensor approach in an unsupervised, unprescribed context requires the development of ad hoc tools, which, to the best of the authors' knowledge, have not been attempted to this extent. Mobilise-D will adopt a multidevice and multisensor system approach that includes multiple IMUs located on different parts of the body, pressure insoles and distance sensors. This novel system, called INDIP (inertial module with distance sensors and pressure insoles) [57], is relatively unobtrusive and allows reference DMO data to be recorded in completely unsupervised conditions. The INDIP reference system is proprietary but for each component commercial alternatives are available. We use a commercial medical grade device for the centre of mass position (McRoberts, DynaPort MM+). Hardware and software requirements such as sampling rate will be made available allowing technology vendors to implement equivalent systems.

A technical validation study is under way in 120 participants (healthy older adults, PD, COPD, MS, PFF and chronic heart failure) recruited across 5 different sites in 3 European countries. Participants are required to undergo a series of observations under different conditions:

(1) Prescribed walking tasks under laboratory conditions

(2) Simulated unprescribed tasks of daily living under laboratory conditions

(3) $2.5 \mathrm{~h}$ of continuous recording in the patient's habitual environment (real world)

(4) 7 days of real-world recording

In all conditions, patients wear the commercial IMU at the centre of mass (McRoberts, DynaPort $\mathrm{MM}+$ ) from which DMOs are derived. To assess precision and accuracy, DMOs derived from the novel algorithms are compared to: gold standards (from motion capture systems and INDIP) under laboratory conditions ( 1 and 2); the INDIP system (condition 3 ) in real-world conditions; and a smartphone app for contextualization including anonymised geolocation, indoors versus outdoors and type of terrain in the real world (conditions 3 and 4). Condition 3 will also allow to label and contextualise DMOs. As a result of this validation study, the largest gold standard digital mobility database will be created to develop optimal

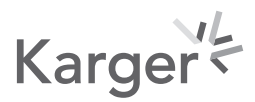


Rochester et al.: The Mobilise-D Approach

DMO algorithms and made available as a reference data set. Detailed algorithm development and benchmarking will be published elsewhere when complete.

Clinical Validation of DMOs: Global Mobility Assessment versus Disease Specificity?

For DMOs to be useful, they need to (i) measure what they aim to measure (i.e., have construct validity), (ii) be related to something that is relevant for the patient (clinically meaningful), clinician or health service (i.e., have reliable predictive ability), and (iii) be able to measure change over time in response to changes in patient characteristics (i.e., sensitive to change). In Mobilise-D we will carry out a clinical validation study to demonstrate that selected DMOs measured with Mobilise-D algorithms have the above-mentioned properties when measured in real life. Clinical validation will be based on a multicentre observational study in 2,400 participants from PD, MS, COPD and PFF cohorts (600 per cohort) recruited in 16 centres across Europe allowing diverse health care systems, geographical areas and different socio-economic regions to be represented. Participants will be followed for 24 months over 5 visits. All participants will wear the commercial IMU (McRoberts, DynaPort $\mathrm{MM}+$ ) which has been validated in the technical validation study for a full 7 days. A detailed protocol and training package is under development to ensure standardised device placement to optimise reliability of data collection.

One important complication is that mobility characteristics and clinical course differ by disease and disease state/stage. It is therefore likely that normative DMOs and the association between DMOs and clinical outcomes are different across diseases. Evaluating DMOs across different disease conditions under a single study protocol is therefore challenging. To address this challenge, we will use 2 approaches to test construct validity, predictive ability and sensitivity to change:

(i) Using disease-specific outcomes, to provide insight into the best DMO for each disease, and where to focus potential future interventions such as the Expanded Disability Status Scale for MS [58] and the Movement Disorders Society - Unified Parkinson's Disease Rating Scale [30]

(ii) Using a disease-independent outcome, such as the Later Life Functional Disability Index [59] and the Short Physical Performance Battery [25], to inform about the properties of DMOs across a wide range of clinical populations with distinct mobility impairment patterns

Aggregation of continuous data into a single outcome measure or DMO presents a unique challenge. Continuous monitoring is ideal when one wants to measure an event that takes place at a given point of time (e.g., a sudden arrhythmia) or count the number of events during a given period of time (e.g., number of apnoeic episodes during sleep time or number of steps during waking hours). However, summarising mobility characteristics is more difficult. For example, whilst there is agreement that walking speed is a relevant DMO driving interest in assessing "real-world" walking speed, the question remains - what is real world? The key is in defining the level and criteria for data aggregation.

Algorithms that have validated the assessment of walking speed do so at the walking bout (episode of walking of a given minimum number of steps) level [39,44]. Walking speed at the walking bout level is complex because people have many bouts during the day of different durations [44] (from a few seconds for a bout inside the house to several hours for an outdoor stroll) and different characteristics (flat surface/uphill/downhill, indoor/outdoor, linear/ curvilinear, etc.). Criteria to select walking bouts included in daily or weekly summary measures must be defined, for example, only those walking bouts lasting at least x minutes, only those that are outdoors, and so on. Finally, given the variability in walking speed across walking bouts in the same person, one needs to decide which statistic best summarises "realworld" walking speed. For example, if mean or median values are taken, the result could be

\section{Karger'}




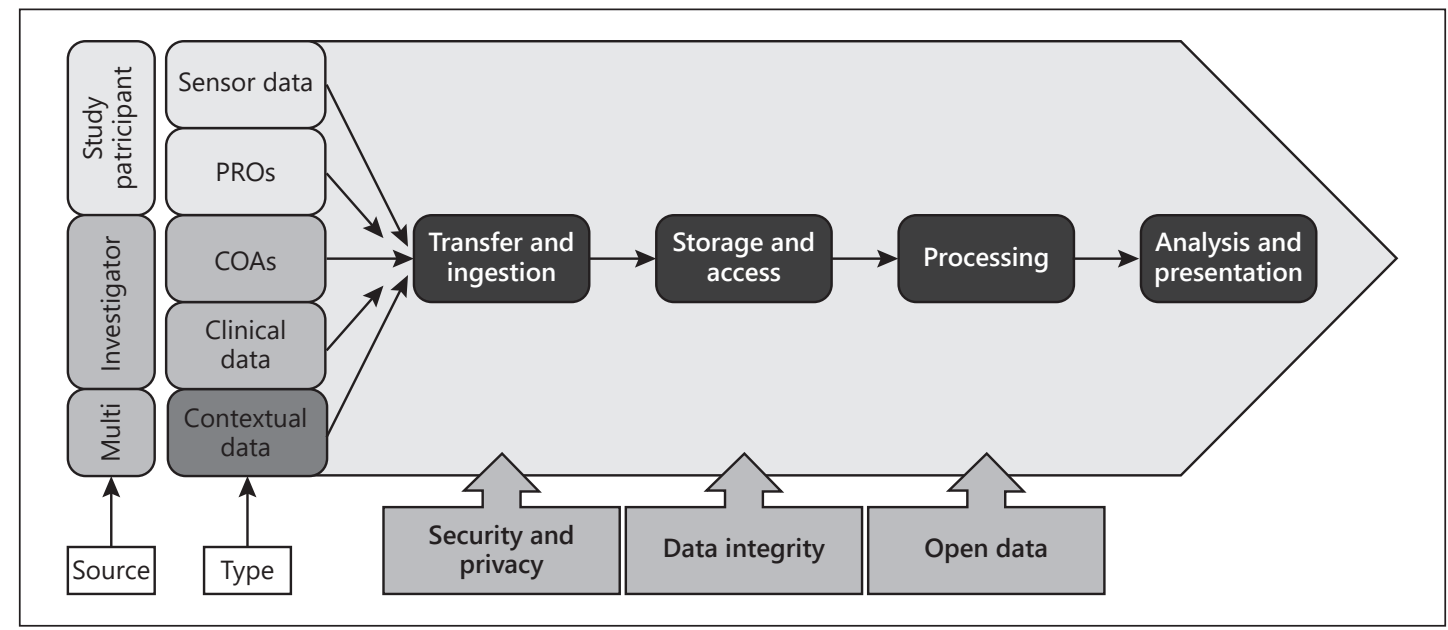

Fig. 2. Mobilise-D data management platform summary. PROs, patient-reported outcomes; COAs, clinical outcome assessments.

influenced by few walking bouts with extreme walking speed. If maximum values are taken, one could argue this is not a measure of walking speed but of exercise capacity. These aspects are being evaluated in Mobilise-D together with other considerations including whether these aspects are similar for all DMOs across cohorts.

\section{Data, Data, Data: Processing, Standards, Security, Ethics}

The Mobilise-D research programme presents a significant data management challenge, and work to address this was started from the outset (Fig. 1). Multidimensional data will be captured across 16 different sites in 10 different countries over multiple measurement periods. In the clinical validation phase alone, we will potentially capture over 2 million hours of sensor data from 2,400 study participants. This requires a standardised approach to process the data (capture, transfer, ingest, integrate, store) prior to analysis (Fig. 2). Standardization must adhere to relevant legal frameworks, and implement appropriate data access and governance models. The data acquired in Mobilise-D will be made available as open data for the research community beyond the term of the research programme, whilst adhering to EU data privacy requirements.

A guiding principle for Mobilise-D is that the highest standards of data integrity are adhered to in all activities. The Mobilise-D consortium has therefore adopted ALCOA+ data integrity principles [60] requiring that all data records are attributable, contemporaneous, original, accurate, complete, consistent, available and enduring. Practically this means that all original source data must be integrated and maintained indefinitely on a secure platform in a manner that preserves the data and privacy rights of the study participants. A rigorous monitoring and quality assurance process ensures data records are accurate and complete and is underpinned by a comprehensive education and support programme for clinical investigators and study participants.

To ensure we meet data privacy and security requirements we are adhering to General Data Protection regulation [61] and directive 2006/24/EC [62] and have implemented a series of privacy-by-design measures to protect the privacy and data rights of study participants throughout the process. All data will be integrated, managed and stored using unique identifier codes. Personal identifiable data will be managed locally and will not be transferred to the central data warehouse. Programme algorithms and data will be stored on e-Science 
Fig. 3. Mobilise-D principles for patient and public engagement, following definitions from NIHR | INVOLVE (https://www.invo.org. $\mathrm{uk} /)$.

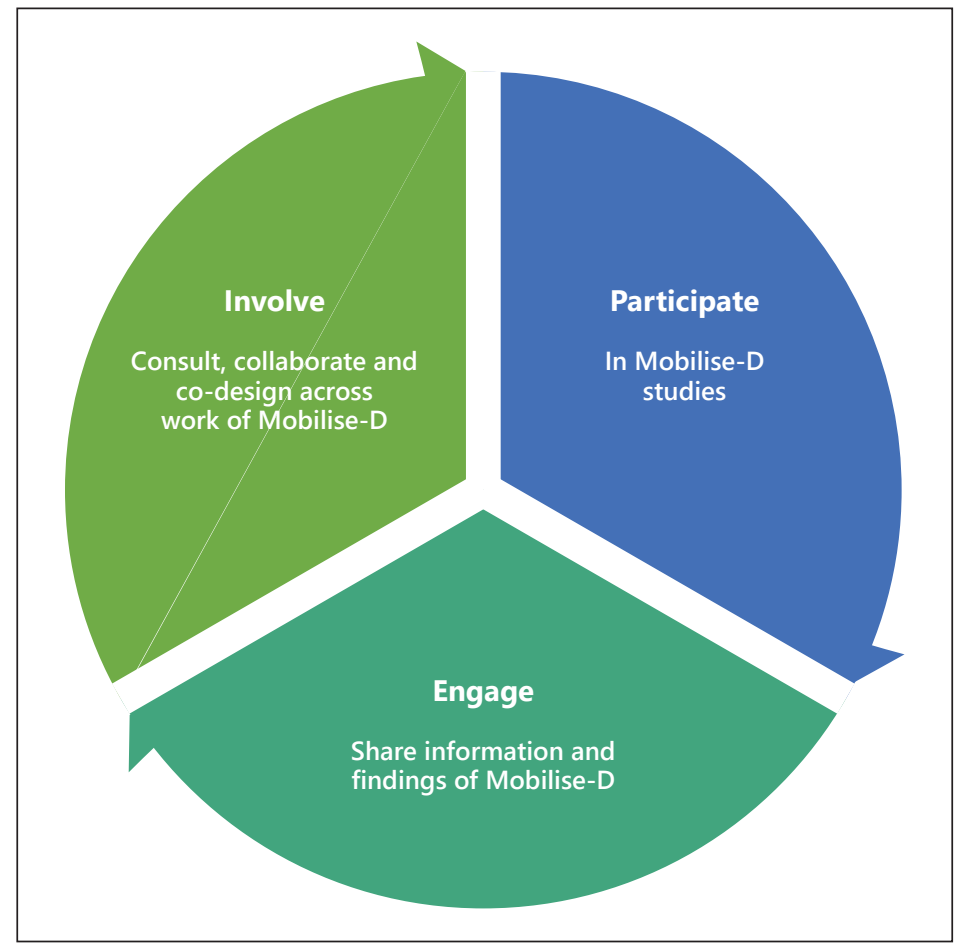

Central, a scalable cloud-based platform to support secure storage, analysis and sharing of multimodal data [54] that is implemented on Amazon Web Services. All data will be encrypted in transit and at rest. Flat files will be stored in S3 buckets which are encrypted using AES-256 encryption. Amazon relational databases will also be encrypted using AES-256. A data warehouse model enables flexibility in application of appropriate analytical workflows.

An access and governance model ensures data are available to appropriate stakeholders when needed during Mobilise-D. Finally, steps to make the data set open and available to the wider research community will ensure ongoing development opportunity beyond Mobilise-D.

\section{Engaging Stakeholders}

An endeavour with the scope and ambition of Mobilise-D invariably draws attention from many interested parties, collectively called stakeholders. Centre stage stakeholders for Mobilise-D are the patients themselves, both current and future, who suffer from mobility loss. From here the circle widens to caregivers, users and providers of health and social care services, patient organisations, industry and academia, as well as the wider public. To ensure meaningful, continued and embedded involvement and engagement of all these stakeholders, Mobilise-D has developed a Patient and Public Involvement and Engagement strategy that details our vision, framework and guiding principles (Fig. 3) to support the integration of patient and public engagement and involvement across the entire process of research, development and validation, and engagement with regulatory agencies, health technology assessment bodies, ethics committees, research organisations and pharmaceutical industries and associations. From the outset, Mobilise-D has engaged the public through frequent updates about the work of the consortium (Fig. 1). Going forward, patients are directly involved in Mobilise-D as participants in our technical and clinical validation studies, and both patients and the public are actively involved through a variety of activities such as 
consultation, collaboration and coproduction of, for example, participant-facing documents and dissemination materials. Mobilise-D has structured patient and public engagement around 5 areas of engagement: (1) discussions at the overall project level, focused on general Mobilise-D approaches and strategies; (2) discussions about digital health technology in general and digital mobility assessment and outcomes in particular; (3) discussions about the usability, value and impact of DMOs in daily life and how the latter can contribute to improved patient care; (4) consultation around health technology assessment and regulatory acceptance of DMOs for clinical use; and (5) involvement in promotion activities about the impact and benefits of Mobilise-D results.

\section{Conclusion}

So what is next? Mobilise-D describes a roadmap outlining the effort needed to bring digital biomarkers from concept to approval. When combined with continuous learning, disease modelling and enhanced methods for data transfer, we predict the benefits, scaling up and implementation will continue to be refined and accelerate the adoption of digital outcomes as routine in research. Similar efforts directed to routine health care will ensure these benefits can be realised for patients with transformation of patient management. Ultimately, the knowledge gained from continuous real-world digital monitoring will provide insights previously unavailable and lead to innovation in therapeutic development and personalised health care.

\section{Acknowledgements}

Claudia Mazzà is supported by the NIHR Sheffield Biomedical Research Centre (BRC). The views expressed are those of the author(s) and not necessarily those of the NHS, the NIHR or the Department of Health and Social Care (DHSC). ISGlobal acknowledges support from the Spanish Ministry of Science and Innovation through the "Centro de Excelencia Severo Ochoa 2019-2023" Program (CEX2018-000806-S) and support from the Generalitat de Catalunya through the CERCA Program.

\section{Statement of Ethics}

No ethical considerations for the publication were necessary.

\section{Conflict of Interest Statement}

L.R. receives research funding from NIHR, EU, MRC, Stroke Association, Parkinsons UK, Cure Parkinsons Trust, Dunhill Medical Trust, Health Research Council, New Zealand. She has served on Ad Board for Biogen. A.A.A. is employee of EMD Serono (a business of Merck KGaA, Darmstadt, Germany). C.B. has received speaker honoraria from Amgen, Pfizer and Nutricia. He has received fees for consultation for E. Lilly on the development of myostatin inhibitors. He has been consulting Philips Health Care/Germany, Bosch Health Care on usage of IMUs and PERS in health care. J.G.-A. reports payments for consulting and lecture fees to her institution from AstraZeneca and lecture fees from Esteeve, Chiesi and Menarini outside the submitted work.

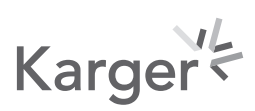


Rochester et al.: The Mobilise-D Approach

\section{Funding Sources}

The MOBILISE-D project has received funding from the Innovative Medicines Initiative 2 Joint Undertaking under grant agreement No. 820820. This Joint Undertaking receives support from the European Union's Horizon 2020 research and innovation programme and the European Federation of Pharmaceutical Industries and Associations (EFPIA).

\section{Author Contributions}

All authors contributed to the overall concept, design, drafting and revision of the paper.

\section{References}

1 Richardson SJ, Carroll CB, Close J, Gordon AL, O’Brien J, Quinn TJ, et al. Research with older people in a world with COVID-19: identification of current and future priorities, challenges and opportunities. Age Ageing. 2020 Oct 23;49(6):901-906.

2 Onder G, Rezza G, Brusaferro S. Case-Fatality Rate and Characteristics of Patients Dying in Relation to COVID-19 in Italy. JAMA. 2020 May;323(18):1775-6.

3 Studenski S, Perera S, Patel K, Rosano C, Faulkner K, Inzitari M, et al. Gait speed and survival in older adults. JAMA. 2011 Jan;305(1):50-8.

4 Fritz S, Lusardi M. White paper: "walking speed: the sixth vital sign”. J Geriatr Phys Ther. 2009;32(2):46-9.

5 Boehme P, Hansen A, Roubenoff R, Scheeren J, Herrmann M, Mondritzki T, et al. How soon will digital endpoints become a cornerstone for future drug development? Drug Discov Today. 2019 Jan;24(1):16-9.

6 Mantua V, Arango C, Balabanov P, Butlen-Ducuing F. Digital health technologies in clinical trials for central nervous system drugs: an EU regulatory perspective. Nat Rev Drug Discov. 2020; DOI: 10.1038/d41573-02000168-z.

7 FDA-NIH Biomarker Working Group. BEST (Biomarkers, EndpointS, and other Tools) Resource. US: Food and Drug Administration; 2016.

8 Coran P, Goldsack JC, Grandinetti CA, Bakker JP, Bolognese M, Dorsey ER, et al. Advancing the Use of Mobile Technologies in Clinical Trials: Recommendations from the Clinical Trials Transformation Initiative. Digit Biomark. 2019 Nov;3(3):145-54.

9 Babrak LM, Menetski J, Rebhan M, Nisato G, Zinggeler M, Brasier N, et al. Traditional and Digital Biomarkers: Two Worlds Apart? Digit Biomark. 2019 Aug;3(2):92-102.

10 Goldsack JC, Coravos A, Bakker JP, Bent B, Dowling AV, Fitzer-Attas C, et al. Verification, analytical validation, and clinical validation (V3): the foundation of determining fit-for-purpose for Biometric Monitoring Technologies (BioMeTs). NPJ Digit Med. 2020;3:55.

11 Badawy R, Hameed F, Bataille L, Little MA, Claes K, Saria S, et al. Metadata concepts for advancing the use of digital health technologies in clinical research. Digit Biomark. 2019 0ct;3(3):116-32.

12 Coravos A, Goldsack JC, Karlin DR, Nebeker C, Perakslis E, Zimmerman N, et al. Digital Medicine: A Primer on Measurement. Digit Biomark. 2019 May;3(2):31-71.

13 Cerreta F, Ritzhaupt A, Metcalfe T, Askin S, Duarte J, Berntgen M, et al. Digital technologies for medicines: shaping a framework for success. Nat Rev Drug Discov. 2020 Sep;19(9):573-4.

14 European Medicine Agency: Questions and answers: Qualification of digital technology-based methodologies to support approval of medicinal product; 2020.

15 Walton MK, Cappelleri JC, Byrom B, Goldsack JC, Eremenco S, Harris D, et al. Considerations for development of an evidence dossier to support the use of mobile sensor technology for clinical outcome assessments in clinical trials. Contemp Clin Trials. 2020 Apr;91:105962.

16 Polhemus AM, Bergquist R, Bosch de Basea M, Brittain G, Buttery SC, Chynkiamis N, et al. Walking-related digital mobility outcomes as clinical trial endpoint measures: protocol for a scoping review. BMJ Open. 2020 Jul;10(7):e038704.

17 Takakusaki K. Functional neuroanatomy for posture and gait control. J Mov Disord. 2017 Jan;10(1):1-17.

18 Yang L, Lu K, Forsman M, Lindecrantz K, Seoane F, Ekblom Ö, et al. Evaluation of physiological workload assessment methods using heart rate and accelerometry for a smart wearable system. Ergonomics. 2019 May; 62(5):694-705.

19 Perera S, Patel KV, Rosano C, Rubin SM, Satterfield S, Harris T, et al. Gait speed predicts incident disability: a pooled analysis. J Gerontol A Biol Sci Med Sci. 2016 Jan;71(1):63-71.

20 Ekelund U, Tarp J, Steene-Johannessen J, Hansen BH, Jefferis B, Fagerland MW, et al. Dose-response associations between accelerometry measured physical activity and sedentary time and all cause mortality: systematic review and harmonised meta-analysis. BMJ. 2019 Aug;366:14570. 
21 Butland RJ, Pang J, Gross ER, Woodcock AA, Geddes DM. Two-, six-, and 12-minute walking tests in respiratory disease. Br Med J (Clin Res Ed). 1982 May;284(6329):1607-8.

22 Bloem BR, Marinus J, Almeida Q, Dibble L, Nieuwboer A, Post B, et al.; Movement Disorders Society Rating Scales Committee. Measurement instruments to assess posture, gait, and balance in Parkinson's disease: critique and recommendations. Mov Disord. 2016 Sep;31(9):1342-55.

23 Motl RW, Cohen JA, Benedict R, Phillips G, LaRocca N, Hudson LD, et al.; Multiple Sclerosis Outcome Assessments Consortium. Validity of the timed 25-foot walk as an ambulatory performance outcome measure for multiple sclerosis. Mult Scler. 2017 Apr;23(5):704-10.

24 Podsiadlo D, Richardson S. The timed "Up \& Go": a test of basic functional mobility for frail elderly persons. J Am Geriatr Soc. 1991 Feb;39(2):142-8.

25 Guralnik JM, Simonsick EM, Ferrucci L, Glynn RJ, Berkman LF, Blazer DG, et al. A short physical performance battery assessing lower extremity function: association with self-reported disability and prediction of mortality and nursing home admission. J Gerontol. 1994 Mar;49(2):M85-94.

26 Lord S, Godfrey A, Galna B, Mhiripiri D, Burn D, Rochester L. Ambulatory activity in incident Parkinson's: more than meets the eye? J Neurol. 2013 Dec;260(12):2964-72.

27 Koolen EH, van Hees HW, van Lummel RC, Dekhuijzen R, Djamin RS, Spruit MA, et al. "Can do" versus "do do": a novel concept to better understand physical functioning in patients with chronic obstructive pulmonary disease. J Clin Med. 2019 Mar;8(3):340.

28 Warmerdam E, Hausdorff JM, Atrsaei A, Zhou Y, Mirelman A, Aminian K, et al. Long-term unsupervised mobility assessment in movement disorders. Lancet Neurol. 2020 May;19(5):462-70.

29 van Lummel RC, Walgaard S, Pijnappels M, Elders PJ, Garcia-Aymerich J, van Dieën JH, et al. Physical Performance and Physical Activity in Older Adults: Associated but Separate Domains of Physical Function in Old Age. PLoS One. 2015 Dec;10(12):e0144048.

30 Goetz CG, Tilley BC, Shaftman SR, Stebbins GT, Fahn S, Martinez-Martin P, et al.; Movement Disorder Society UPDRS Revision Task Force. Movement Disorder Society-sponsored revision of the Unified Parkinson's Disease Rating Scale (MDS-UPDRS): scale presentation and clinimetric testing results. Mov Disord. 2008 Nov; 23(15):2129-70.

31 Hobart JC, Riazi A, Lamping DL, Fitzpatrick R, Thompson AJ. Measuring the impact of MS on walking ability: the 12-Item MS Walking Scale (MSWS-12). Neurology. 2003 Jan;60(1):31-6.

32 Gimeno-Santos E, Raste Y, Demeyer H, Louvaris Z, de Jong C, Rabinovich RA, et al.; PROactive consortium. The PROactive instruments to measure physical activity in patients with chronic obstructive pulmonary disease. Eur Respir J. 2015 Oct;46(4):988-1000.

33 Taraldsen K, Vereijken B, Thingstad P, Sletvold O, Helbostad JL. Multiple days of monitoring are needed to obtain a reliable estimate of physical activity in hip-fracture patients. J Aging Phys Act. 2014 Apr;22(2):173-7.

34 Donaire-Gonzalez D, Gimeno-Santos E, Balcells E, Rodríguez DA, Farrero E, de Batlle J, et al. Physical activity in COPD patients: patterns and bouts. Eur Respir J. 2013 Oct;42(4):993-1002.

35 European Medicine Agency. Qualification opinion on stride velocity 95th centile as a secondary endpoint in Duchenne Muscular Dystrophy measured by a valid and suitable wearable device (2019). Available from: https://www.ema.europa.eu/en/documents/scientific-guideline/qualification-opinion-stride-velocity95th-centile-secondary-endpoint-duchenne-muscular-dystrophy_en.pdf.

36 European Medicine Agency. Qualification Opinion on Proactive in COPD, 2018. Available from: https://www. ema.europa.eu/en/documents/regulatory-procedural-guideline/qualification-opinion-proactive-chronicobstructive-pulmonary-disease-copd_en.pdf.

37 U.S. Food \& Drug Administration. Review of the letter of intent (LOI) submission for DDT COA \# 000106, 2018. Available from: https://www.fda.gov/media/124846/download.

38 Viceconti M, Hernandez Penna S, Dartee W, Mazzà C, Caulfield B, Becker C, et al. Toward a Regulatory Qualification of Real-World Mobility Performance Biomarkers in Parkinson's Patients Using Digital Mobility Outcomes. Sensors (Basel). 2020 Oct;20(20):E5920.

39 Del Din S, Godfrey A, Mazzà C, Lord S, Rochester L. Free-living monitoring of Parkinson's disease: lessons from the field. Mov Disord. 2016 Sep;31(9):1293-313.

40 Aminian K, Najafi B, Büla C, Leyvraz PF, Robert P. Spatio-temporal parameters of gait measured by an ambulatory system using miniature gyroscopes. J Biomech. 2002 May;35(5):689-99.

41 Barry G, Galna B, Lord S, Rochester L, Godfrey A. Defining ambulatory bouts in free-living activity: impact of brief stationary periods on bout metrics. Gait Posture. 2015 Oct;42(4):594-7.

42 El-Gohary M, Pearson S, McNames J, Mancini M, Horak F, Mellone S, et al. Continuous monitoring of turning in patients with movement disability. Sensors (Basel). 2013 Dec;14(1):356-69.

43 Haji Ghassemi N, Hannink J, Roth N, Gaßner H, Marxreiter F, Klucken J, et al. Turning Analysis during Standardized Test Using On-Shoe Wearable Sensors in Parkinson's Disease. Sensors (Basel). 2019 Jul;19(14): 3103-14.

44 Del Din S, Godfrey A, Galna B, Lord S, Rochester L. Free-living gait characteristics in ageing and Parkinson's disease: impact of environment and ambulatory bout length. J Neuroeng Rehabil. 2016 May;13(1):46.

45 Storm FA, Buckley CJ, Mazzà C. Gait event detection in laboratory and real life settings: accuracy of ankle and waist sensor based methods. Gait Posture. 2016 Oct;50:42-6.

46 Lee H, You J, Cho S, Hwang S, Lee D, Kim Y, et al. Computational methods to detect step events for normal and pathological gait evaluation using accelerometer. Electron Lett. 2010;46(17):1185-7. 
47 Ull[i]rich M, Hannink J, Gaßner H, Klucken J, Eskofier B, Kluge F. Unsupervised harmonic frequency-based gait sequence detection for Parkinson's disease. IEEE EMBS International Conference on Biomedical \& Health Informatics (BHI). 2019:1-4.

48 Kheirkhahan M, Chen Z, Corbett D, Wanigatunga A, Manini T, Ranka S. Adaptive walk detection algorithm using activity counts. IEEE EMBS International Conference on Biomedical \& Health Informatics (BHI) 2017:161164.

49 Chigateri NG, Kerse N, Wheeler L, MacDonald B, Klenk J. Validation of an accelerometer for measurement of activity in frail older people. Gait Posture. 2018 Oct;66:114-7.

50 Hickey A, Del Din S, Rochester L, Godfrey A. Detecting free-living steps and walking bouts: validating an algorithm for macro gait analysis. Physiol Meas. 2017 Jan;38(1):N1-15.

51 Trojaniello D, Cereatti A, Pelosin E, Avanzino L, Mirelman A, Hausdorff JM, et al. Estimation of step-by-step spatio-temporal parameters of normal and impaired gait using shank-mounted magneto-inertial sensors: application to elderly, hemiparetic, parkinsonian and choreic gait. J Neuroeng Rehabil. 2014 Nov;11(1):152.

52 Fasel B, Spörri J, Schütz P, Lorenzetti S, Aminian K. An Inertial Sensor-Based Method for Estimating the Athlete's Relative Joint Center Positions and Center of Mass Kinematics in Alpine Ski Racing. Front Physiol. 2017 Nov; 8:850.

53 Micó-Amigo ME, Kingma I, Ainsworth E, Walgaard S, Niessen M, van Lummel RC, et al. A novel accelerometrybased algorithm for the detection of step durations over short episodes of gait in healthy elderly. J Neuroeng Rehabil. 2016 Apr;13(1):38.

54 Hiden H, Woodman S, Watson P, Cala J. Developing cloud applications using the e-Science Central platform. Philos Trans A Math Phys Eng Sci. 2012 Dec;371(1983):20120085.

55 Bourke AK, Ihlen EA, Bergquist R, Wik PB, Vereijken B, Helbostad JL. A physical activity reference data-set recorded from older adults using body-worn inertial sensors and video technology-The ADAPT study data-set. Sensors (Basel). 2017 Mar;17(3):17.

56 Buckley C, Alcock L, McArdle R, Rehman RZ, Del Din S, Mazzà C, et al. The role of movement analysis in diagnosing and monitoring neurodegenerative conditions: insights from gait and postural control. Brain Sci. 2019 Feb;9(2):34.

57 Bertuletti S, Salis F, Cereatti A, Angelini L, Buckley E, Nair KP, et al. Inter-leg Distance Measurement as a Tool for Accurate Step Counting in Patients with Multiple Sclerosis. Annu Int Conf IEEE Eng Med Biol Soc. 2019 Jul; 2019:6413-7.

58 Kurtzke JF. Rating neurologic impairment in multiple sclerosis: an expanded disability status scale (EDSS). Neurology. 1983 Nov;33(11):1444-52.

59 Jette AM, Haley SM, Coster WJ, Kooyoomjian JT, Levenson S, Heeren T, et al. Late life function and disability instrument: I. Development and evaluation of the disability component. J Gerontol A Biol Sci Med Sci. 2002 Apr;57(4):M209-16.

60 World Health Orgnaisation: Guidelines on good data and record management practices; 2018.

61 European Parliament: Regulation (EU) 2016/679 of the European Parliament and of the Council on the protection of natural persons with regard to the processing of personal data and on the free movement of such data, and repealing Directive 95/46/EC (General Data Protection Regulation); 2016.

62 European Parliament: Directive 2006/24/EC of the European Parliament and of the Council on the retention of data generated or processed in connection with the provision of publicly available electronic communications services or of public communications networks and amending Directive 2002/58/EC; 2006. 